\title{
¿Evolución o revolución? Análisis de opinión y de percepciones por parte de los principales agentes transformadores de la cultura green en el ámbito del coche eléctrico en Londres y Barcelona
}

\author{
Enric ORDEIX \\ Universidad Ramon Llull \\ enricor@blanquerna.url.edu \\ Josep RoM \\ Universidad Ramon Llull \\ joseprr@blanquerna.url.edu \\ Jordi BoteY \\ Universidad Ramon Llull \\ jordibl@blanquerna.url.edu
}

\begin{abstract}
Resumen:
La presente investigación pretende colaborar en la definición de nuevas políticas de comunicación para el uso y desarrollo de la movilidad eléctrica en cooperación con universidades de Barcelona, Londres y Milton Keynes (Inglaterra) y empresas y administraciones de ambos países. En una primera fase se han investigado los agentes que contribuyen al cambio -sus percepciones y motivaciones-, y, en una segunda fase los efectos que han tenido sus opiniones en al opinión pública. Según lo estudiado podemos establecer los siguientes resultados: en relación a las nuevas estructuras comunicativas, según lo establecido por las conocidas teorías sistémicas, vemos que crece la sinergia social, en inglés Grassroots (Payne, 2009), en relación a la acción participativa de los nuevos sistema de movilidad entendidos como sostenibles que subyacen en la Cultura Green. Percibimos la demanda evidente por parte de los colectivos sociales que de alguna manera 'obligan' o inducen a las grandes empresas productores de coches eléctricos a adoptar nuevas políticas de desarrollo sostenible y/o de compromiso social (David et al., 2005) en que exista una actuación activa por parte de ciudadano y empresa. Percibimos una forma nueva de actuar para y con los públicos, en tanto que se pide mayor implicación y más proacción no sólo en la forma de consumir, sino en las nuevas formas de actuar ante la sociedad. Este concepto nos lleva al del "Shared Interest" entre consumidor y empresa, concepto evolucionado de uno de los preceptos establecidos en por Grunig (2009) a través de la idea de diálogo y de la simetría comunicativa. En muchos mercados, especialmente en el inglés, los grupos de opinión están pidiendo una mayor implicación de la comunidad a través de las entidades sociales, y de los grupos de interés en el desarrollo de productos menos dañinos con el medioambiente. Este activismo social positivo a favor de las empresas sensibles a los valores sociales está causando una opinión a priori positiva por parte de los varios stakeholders, líderes de opinión o agentes de cambio.
\end{abstract}

Palabras clave: Agente social de cambio; cultura Green; vehículo eléctrico; relaciones públicas; transporte sostenible 


\title{
Evolution or revolution? Analysis of Perceptions and Opinion by Main Green Culture Transformer Agents in the Electric Cars Industry in London and Barcelona
}

\begin{abstract}
:
The current research wants to approach the reader to the definition of new communication policies in the usage and development of electric mobility in cooperation with universities of Barcelona, London and Milton Keynes (England) as well as companies and public administration from both countries. The first phase of the research was focused on describing the transformation agents- its perspectives and motivations-. The second phase of the research was to detect this agent's influence in the public opinion. The results of the research were the following: the new communication structures, as is stated in the systemic theory, social synergy, also called Grassroots (Payne, 2009), is growing so consistently specially because the collective participation in setting the new mobility system within the framework of the sustainability an the incoming Green Culture. We notice a new demand by society that drive big companies that produce electric cars. We Perceive the obvious demand from social groups that somehow 'force " or induce large companies electric car producers to adopt new sustainability and development policies in order to get social engagement (David et al., 2005 ) We perceive a new way of communicating "for and with the public", while a higher praction is required due to new ways of social consumption. This leads us to the concept of "Shared Interest" between consumer and organizations, precept established by Grunig (2009) through the idea of dialogue and communicative symmetry. In many markets, especially in English, social groups are calling for a greater community involvement through social organizations, in order to develop less environmentally harmful products. This social activism is positively sensitive to corporate values as well sensitive to the society values and creates a positive opinion towards opinion leaders and other kind of stakeholders that act as changing society agents.

Electric vehicles suddenly appear as an evolution of the automotive industry and a sign of the revolution of the environmental polices.
\end{abstract}

Key Words: Green Culture; Social Transformer Agent; Electric Vehicle; Public Relations; Sustainable Transportation

\section{Referencia normalizada:}

Ordeix, E., Rom, J. y Botey, J. (2014): ¿Evolución o Revolución? Análisis de opinión y de percepciones por parte de los principales agentes transformadores de la cultura green en el ámbito del coche eléctrico en Londres y Barcelonas. Historia y Comunicación Social. Vol. 19. Núm. Especial Marzo. Págs. 819-830.

Sumario: 1. Introdución. 2. Metodología. 3. Resultados. 4. Conclusiones.

\section{Introducción}

Los autores consideran esta temática está asociada a la creciente cultura Green, de creciente interés por varias razones. A nivel mundial, se estima en las grandes ciudades ya son unas 180.000 personas las que se trasladan en coche eléctrico. Esté número va a aumentar exponencialmente si se cumplen dos previsiones: uno, que la cuota de mercado de vehículo eléctrico se mantenga esencialmente en el ámbito urbano; y, dos, que el número de habitantes de las grandes ciudades siga creciendo 
hasta los 6,4 mil millones en 2050. Si tenemos en cuenta el informe del Banco Mundial Transforming Cities with Transit (Potter et al, 2011) donde se afirma que la eficiencia del transporte es indispensable para la vitalidad económica de la ciudad y que en los países desarrollados el principal método de transporte ha sido el automóvil personal, podemos estimar que unos 2,3 mil millones adicionales de coches saldrán a las carreteras de aquí a 2050. China proyecta tener más de 900 millones de vehículos motorizados para el año 2050.

Los vehículos eléctricos aparecen de repente como una forma de evolución de la industria del automóvil que para algunos tiene el aire de una verdadera revolución de las políticas del medio ambiente que obedece a nuevas respuestas sociales al uso de una tipología ya obsoleta de automóviles. Sin embargo, nos enfrentamos a limitaciones tecnológicas y de aceptación de uso por parte de los usuarios (menos autonomía y velocidad, más duración en la recarga, entre otros) que, a su vez, conlleva connotaciones ideológicas y simbólicas que obedecen, como hemos dicho, a la creciente cultura green en los medios de transporte (Ingenhoff; Fuhrer, 2010). Así pues, los investigadores de este estudio consideramos que uno de los principales retos de esta transformación social es la definición de las políticas de divulgación de la nueva cultura de la movilidad eléctrica en las ciudades, especialmente en las llamadas ciudades Smart, como Londres y Barcelona.

\section{Metodología}

La metodología se ha basado en una serie de entrevistas y discusiones en dinámicas de grupo en tres exploratory workshops (Daymon y Holloway, 2002) a actores y colectivos del sector (empresarios del sector de la automoción y la energía, técnicos de transporte, miembros de la administración pública e investigadores universitarios) llevados a cabo físicamente en tres universidades y ciudades diferentes: la Open University (Milton Keynes), la Universitat Ramon Llull (Barcelona) y la Greenwich University (Londres). El estudio es fruto de una investigación sufragada por el Banco Santander - por la rama inglesa-y por el Grupo de Investigación en Estrategia y Creatividad en Publicidad y Relaciones Públicas de la URL- por la rama española. Las entrevistas se realizaron siguiendo una guía de entrevista (Holtzhausen, Voto, 2002) basada en los indicadores que mencionamos a continuación.

Los aspectos considerados en el análisis han sido los correspondientes a los principios establecidos en "Constructing The Social Personality in Organizations" (Ordeix, 2014), tales como la legitimidad, el liderazgo de opinión, el compromiso social y la capacidad de establecer sinergias sociales a partir del diálogo. Y, a su vez, también los inspirados en los parámetros descritos en el estudio "Excellence in Public Relations and Communication Management" dirigido por James Grunig, Larisa Grunig y David Dozier (2002), tales como el sistema organizativo, la estructura de la organización, la presencia pública y la priorización y proactividad comunicativa. Su inter- 
pretación y concreción ha sido elaborada en anteriores estudios (Ordeix; Rom; Botey, 2013), a través de los cuales se han establecido los parámetros que nos sirven ahora para analizar la evolución de los agentes de cambio y parte de su comunicación en la implantación del vehículo eléctrico en Barcelona y Londres, en tanto que ciudades muestra.

En la metodología se han establecido cuatro indicadores, para analizar el cambio de opinión y las percepciones públicas. Estos indicadores les dan capacidad de crear agenda a las organizaciones, y por consiguiente, influyen en su identidad y discurso.

El primer concepto que hemos considerado es el sistema de comunicación con tendencia simétrica, según los criterios de la escuela de Maryland (Grunig, 2002), busca del espacio común de intereses, también llamado mutual understanding. La comprensión mutua conlleva un diálogo intenso que en el extremo nos conduce a un control compartido de la actividad comunicativa de la organización, tanto por parte de los que dirigen y lideran la empresa como por parte de los públicos receptores inscritos en la red de públicos del sistema de la organización. También hay que tener en cuenta la crítica de Heath (2000) y de Heath y Cooms (2006), en que añade que los modelos sociales y económicos, actuales inducen al desequilibrio y, por tanto relegan la postura de Grunig (2006) a un mero deseo y modelo idealista.

La estructura organizativa en las empresas es otro indicador a tener en cuenta (Botam; Hazleton, 2005). Las empresas abiertas y permeables al entorno y demandas sociales han contribuido a acelerar el cambio de opinión y han provocado una mayor y más positiva perspectiva de los vehículo o transporte eléctrico en general. Creemos que los departamentos de comunicación y relaciones públicas se han encargado de establecer creando escenarios y experiencias sociales positivas que han ayudado en la captación de tendencias por parte de los productores, y potenciado la canalización de ciertas reivindicaciones por parte de los colectivos con capacidad de crear agenda mediática y política. Entendemos que sólo los intereses compartidos pueden convertirse potencialmente en actividades conjuntas entre las partes. Establecemos tres tipos de vínculos (Grunig, 2002); el comercial (por acuerdos en proyectos concretos), el normativo (por las nuevas normas de la Unión Europea en este ámbito), y el emotivo (como muchas veces se ha atribuido a los desarrollos empresariales afines a la sostenibilidad y la responsabilidad social corporativa, en general).

El tercer parámetro es el que se establece a partir de la teoría situacional de públicos de Grunig (2006). Esta teoría concibe el entorno social como un todo interaccionado y sólo a partir de una concepción holística de la actuación empresarial es posible una actuación perspicaz y efectiva para la creación de opinión y general efectos de cambio real en los mencionados agentes de cambio. Así pues, la complicidad y el compromiso de las grandes productoras del sector del automovilismo con las organizaciones sociales, políticas e institucionales, son un factor determinante para la instauración de nuevas agendas y prioridades en el sector.

A su vez, y a consecuencia de ello han nacido nuevos roles en el terreno de la comunicación y planificación estratégica que priorizan la fidelización a la expansión 
comercial a lo que nos tenían acostumbrados en periodos de auge económico. La teoría situacional de públicos facilita la proyección del discurso persuasivo de la organización (Heath; Cooms, 2006) y abre la puerta a las políticas de responsabilidad social de la misma.

El cuarto y último parámetro establecido es la proactividad y priorización estratégica de la comunicación propia de una la filosofía (la Cultura Green) que ya amaraba de la mayor parte de las grandes productoras del sector, pero que no se aplicaba en su plenitud. Si observamos los valores corporativos de la mayoría de las compañías el discurso Green ya parecía haberse implantado en de hacía tiempo.

Los principios de la excelencia (Ordeix; Rom, 2012) establecen que, una organización que procura por su implicación social y que trabaja por ser concebida como empresa alineada con los valores sociales imperantes. Es a partir de la crisis económica que se ha detectado uno de los resultados menos esperanzadores que ha provocado el tema de la producción eléctrica en la prioridad estratégica, como revulsivo y forma de dinamizar el sector.

En definitiva, a partir de los cuatro indicadores principales, podríamos considerar la estructura de la organización y la teoría situacional de públicos como los conceptos más relevantes y significativos. Aunque existen otros parámetros a considerar, colaterales a los establecidos como son la influencia, la innovación, la capacidad de investigación, el emprendimiento. Cabe añadir que Huertas y Ordeix $(2012,2013)$ establecían otros conceptos y variables a considerar también en nuestro análisis:

- la respuesta del departamento de comunicación y relaciones públicas ante los problemas sociales,

- la participación la empresa en la toma de decisiones importantes y grandes iniciativas del gobierno,

- el apoyo de la dirección general a las actividades que desarrolla el departamento de comunicación y relaciones públicas,

- la creación de oportunidades para que los directivos puedan oír y conozcan las opiniones de los varios actores sociales,

- la representación y presentación de las empresas del sector en eventos y meetings,

- la presencia del sector en los medios de comunicación y su capacidad de crear agenda,

- la influencia del sector en la toma de decisiones políticas,

- la capacidad de crear conocimiento per parte de las empresas del sector en los temas de mayor trascendencia, especialmente en aquellos relativos a la innovación y avances tecnológicos.

Por lo que se refiere a la hipótesis que se plantea aquí afirma que los agentes de cambio asociados a la implantación de las varias tipologías de vehículos eléctricos 
están desarrollando un estado de opinión favorable a su venta y plena comercialización, debido, en parte a una nueva concepción comunicativa: estructura de la comunicación de las organizaciones, nueva incursión pública de éstas y nuevas formas de planificación y priorización de los proyectos de relaciones públicas y de comunicación. Pensamos que esto se sustenta en dos hechos clave: primero, la mayor parte de las grandes marcas a nivel mundial están acabando de perfilar sus prototipos $\mathrm{y}$, a su vez, están empezando a comercializar de forma iniciática sus primeros modelos; y segundo, la bajada de la venta del vehículo clásico de gasolina, hecho que se debe, en gran parte, a un cambio de paradigma en la forma de establecer la movilidad en las grandes urbes, más que sólo a una evidente crisis económica.

\section{Resultados}

De los grupos de discusión realizados por los expertos del sector en los workshops de Milton Keynes, Barcelona y Londres se pueden establecer unas consideraciones generales:

- La planificación de las políticas de transporte inteligente de las ciudades de Barcelona y Londres se diseñan sin destacar el papel de las tecnologías emergentes, como los vehículos eléctricos.

- Los agentes de cambio o líderes de opinión en ambas ciudades coinciden en la necesidad de establecer un puente entre las políticas de movilidad urbana y los vehículos eléctricos. Se constata la sensibilidad de los ciudadanos por un nuevo modelo de movilidad sostenible, pero no se establece un vínculo entre los retos de la movilidad urbana y las oportunidades que aportan los vehículos eléctricos como nuevos medios.

- Los agentes del sector empresarial se encuentran inmersos en un contexto comercial de lanzamiento de nuevos modelos de vehículos eléctricos con una respuesta limitada por parte de los usuarios.

- Los agentes de las administraciones reconocen, en ambas ciudades, una limitación en la divulgación de las políticas de implementación de soluciones para el uso de vehículos eléctricos en las ciudades.

- Los agentes de las universidades -expertos en transporte, tecnología de automoción, políticas de movilidad y comunicación- coinciden en la necesidad de aproximar a la opinión pública los avances y las soluciones que aportan los nuevos medios a un modelo de movilidad urbana sostenible.

El debate en grupos de discusión entorno a estos ejes alcanzó un alto grado de sinergia hasta concretarse en unos temas de interés comunes a todos los agentes de cambio: 
- Diseñar un modelo de transición entre el modelo de transporte tradicional y el modelo de transporte inteligente.

- Definir los nuevos modelos de negocio para las nuevas soluciones de transporte.

- Establecer una asociación entre los agentes de cambio de ambas ciudades para trabajar en el desarrollo de las oportunidades de negocio y nichos de negocio de los vehículos eléctricos apropiadas a las condiciones locales.

- El apoyo a la difusión de las nuevas políticas de transporte y de innovación tecnológica.

- Las implicaciones de los nuevos sistemas de transporte en el desarrollo de una cultura green de movilidad sostenible.

- El despliegue de nuevas estrategias y mejores prácticas para mitigar los problemas de transporte urbano y el desarrollo de soluciones sostenibles de transporte, incluyendo un enfoque particular en los vehículos eléctricos.

Las entrevistas en profundidad, centradas en los indicadores de legitimidad/ sinergia social, liderazgo de opinión, compromiso social y capacidad de establecer sinergias a partir del diálogo permiten establecer coincidencias importantes en los resultados obtenidos en Barcelona y Londres. La amplia mayoría de los entrevistados coinciden en la valoración estratégica de la comunicación como motor de cambio y en la legitimidad de las organizaciones implicadas en la difusión de las soluciones de transporte basadas en los vehículos eléctricos. También percibimos la importancia de los valores de legitimidad, representatividad, experiencia social y consensual, como fundamentos de la identidad de las administraciones y las universidades implicadas. En el sector de la empresa la necesidad de establecer un valor de representatividad social es especialmente intenso debido a las peculiaridades del sector del automóvil. En el caso de España y el Reino Unido las empresas dedicadas al desarrollo de vehículos eléctricos se dividen entre las pequeñas empresas de bicicletas y motocicletas eléctricas y las grandes empresas multinacionales del automóvil con una implantación muy fuerte en el territorio barcelonés. A las pequeñas empresas les cuesta actuar como interlocutores sociales y carecen de medios para mejorar la comunicación y el diálogo con sus usuarios potenciales, además existe un recelo importante entre las empresas especializadas y los grandes grupos de automoción y energía. En el caso del sector universitario, existe una mayor complicidad con las administraciones que permite asumir el liderazgo de opinión del tema ante la opinión pública. En general se constata un desconocimiento de las motivaciones de los usuarios y la voluntad de establecer plataformas de divulgación de la cultura de la movilidad eléctrica basadas en el diálogo entre los agentes de cambio y los usuarios. Si agrupamos la tipología de respuesta a las entrevistas en función de los indicadores básicos -fundamentados en la filosofía investigadora del estudio de la excelencia- podemos percibir claramente que las coincidencias entre los agentes de cambio de Barcelona y Londres son mucho mayores que las diferencias. 
Parece, hasta el momento, que la opinión publicada presentada en los varios foros es muy favorable a la inversión e innovación de la empresa automovilística en la producción de prototipos y comercialización de coches eléctricos. Sin embargo, su pleno desarrollo, todavía está en entredicho.

Nuevos sistemas de liderazgo social. El líder de opinión es el que actúa como verdadero agente de cambio en lo que le llamamos "La Revolución de la Cultura Green" y es el que mejor conecta con los valores de la comunidad. Las variadas instituciones, consorcios entre empresas productoras y administración públicas, así como empresas del sector eléctrico y de la logística -ver el caso de LIVE en Barcelona-, se unen a otros líderes de opinión y analistas crecientes los grupos de investigación frutos de la alianza Londres/Milton Keynes y Barcelona (Greenwich University, Open University y Universitat Ramon Lull), gracias al soporte recibido por parte de grupo Santander. Esta estructura de liderazgo compartido muestra el alto grado de interés público y/o general (Godfrey et al, 2009), pero también la gran reticencia por parte de la industria automovilística a asumir el proyecto de transformación en solitario. Otra vez vemos otros sistemas comunicativos más horizontales y dialogantes y una planificación estratégica muy detallada, con una ejecución muy cautelosa. La búsqueda de la legitimidad para actuar y representar (Ordeix, Duarte, 2008) el "Movimiento Green" a través de la ya inminente comercialización masiva de coches eléctricos es un hecho.

Efectividad y diversidad en nuevos canales comunicativos. La creación de núcleos de opinión en grupos ha provocada, por lo general, una publicity positiva, cosa que nos hace entrever que la creación de opinión al respecto es ya un hecho. A su vez el buzz en los popularmente denominados new media tiene unos efectos de contención en el consumidor potencial que puede provocar efectos en un potencial, ya casi inminente, lanzamiento de los productos de movilidad eléctrica, ya sean bicicletas, motocicletas, como coches u otros medios de locomoción (Botey; Martin; Rom, 2013). Así pues vemos nuevas estructuras empresariales y nuevos planteamientos comunicativos más transversales y holísticos a la búsqueda de la excelencia y máxima efectividad en la búsqueda de una opinión y de un máximo soporte social.

\section{Conclusiones}

Los resultados del estudio se pueden sintetizar en dos objetivos comunes adoptados por todos los agentes participantes: potenciar la comprensión del comportamiento de los usuarios para establecer estrategias de divulgación más eficientes en lugar de centrarse en la comunicación de los conocimientos tecnológicos, y sintetizar y compartir la información y la investigación sobre la movilidad sostenible para mejorar la percepción social de los vehículos eléctricos.

A) Participación : Obtener grupos destinatarios activos 
En las cuestiones ecológicas los activistas actúan como impulsores de determinantes políticas corporativas, así cómo de innovaciones y dinámicas de la industria del sector automovilístico. La "Cultura Green" está provocando nuevas dinámicas organizativas y de comunicación nuevas en el sector (Botey; Martin; Rom, 2013) y que están afectando, en conjunto, a la comunicación de toda la línea de productos. A su vez, la comunicación es más bien consorciada y cooperada con la administración y otras industrias paralelas como la de la electricidad, la logística y la informática. Como hemos dicho, la implantación de la movilidad eléctrica lleva implícita una nueva política comunicativa más holística y transversal y/o horizontal con los stakeholders, que a su vez participan de las innovaciones y dinámicas en proyectos de investigación y desarrollo (Xifra; Ordeix, 2009). Vemos ejemplo de ello en los grandes proyectos interuniversitarios en los que hemos participado, o bien el las muestras de prototipos en entornos peculiares como pequeñas ciudades o barrios.

B) Transversalidad es gestión del conocimiento.

El conjunto del proyecto comunicativo de participación está estrechamente vinculado a las herramientas de gestión del conocimiento (Lalueza, 2006). Esto nos conduce al convencimiento que las partes implicadas en la ya mencionada "Revolución Green" buscan que su interés particular impulse un interés común, muy alineado con la nueva concepción de las ciudades inteligentes - a menudo llamadas Smart Cities- (Simó et al., 2012). Así pues, el compromiso social o colectivo de los distintos sectores que intervienen no dan lugar a un tipo de comunicación de tipo más comercial, pero sí institucional y con liderazgo compartido, tanto por lo que se refiere a Londres como a Barcelona.

\section{C) La legitimidad y el medio plazo}

La industria automovilística, las eléctricas, el sector tecnológico y logístico, así como la administración pública de ambos países están esperando que los grupos influentes de la sociedad se activen y actúen como verdaderos actores de cambio social. El conocido social engagement es de vital importancia en el proceso comunicativo institucional (Ordeix, Duarte, 2009). La influencia de base o soporte social es de suma relevancia, como para vencer los agravios comparativos con el uso del coche convencional, y poder potenciar su producción en serie. Para medir el soporte, requeriríamos de mapas de influencia social, con sus grupos participantes y actitudes compartidas. En nuestro estudio, todavía hay que ampliar con detalle este aspecto.

Así pues podemos decir que este proyecto nos ha permitido explorar tres aspectos diferenciados por lo que se refiere a la cultura e implantación/conocimieto del Vehículo Eléctrico:

Mensaje inclusivo. Vemos que las empresas automovilísticas y las administraciones están haciendo un esfuerzo para integrar el proyecto de desarrollo del vehículo eléctrico a un entorno de interés general, creando insights asociados a la nueva concepción de las Smart Cities. 
Percepción de inmadurez. Las opiniones percibidas en los varios workshops y seminarios exploratorios, con líderes de opinión académicos, productores e ingenieros de tecnología y logística, son de que el sector quiere pero no se siente preparado, a la vez que no está maduro como para ser competitivo. Esta percepción impide que se puedan llevar a término las verdaderas transformaciones sociales y económicas que esto implica en tanto que nuevo sistema de movilidad.

El liderazgo compartido frena su desarrollo. Aunar objetivos e intereses, así como valores y actitudes, es sumamente dificultoso en procesos de transformación sumamente radical como este. No sólo por lo que se refiere a las nuevas estructuras empresariales y de cooperación institucional, sino también por la necesidad de adaptar sistemas de comunicación y relaciones públicas más participativos, dialogantes (Toledano, Mckie, 2007) y con visión amplia de los públicos de influencia.

Por último, cabe añadir que convendría las distintas empresas y administraciones consorciadas implicadas en esta transformación delimitasen su scope o ámbito de influencia deseado en este proyecto, pues evitaría posibles crisis ante la amplia expectativa de cambio que está generando este tema en muchas de las grandes ciudades europeas.

\section{Bibliografía}

BOTAM, C.; HAZLETON, V. (2006). Public Relations Theory. Lawrence Erlbaum. Mahwah, NJ.

DAVID, P.; KLINE, S.; DAI, Y. (2005). "Corporate social responsibility practices, corporate identity and purchase intention: a dual-process model". En: Journal of Public Relations Research, 17, 3, 291-313.

DAYMON, C.; HOLLOWAY, I. (2002). Qualitative Research Methods in Public Relations and Marketing Communications. Routledge, Milton Park, Oxon, UK.

GODFREY, P. C.; MERRILL, C. B.; HANSEN, J. M. "The relationship between corporate social responsibility and shareholder value: An empirical test of the risk management hypothesis". En: Strategic Management Journal, 30, 2009, p. 425-445.

GRUNIG, L.; GRUNIG, J.; DOZIER, D. M. (2002). Excellent public relations and effective organizations: A study of communication management in three countries. Lawrence Erlbaum, Mahwah, US.

GRUNIG, J. (2006). Furnishing the Edifice: Ongoing Research on Public Relations As a Strategic Management Function. En: Journal of Public Relations Research, 18 (2), 2006, p. 151-176.

HEATH, R.L.; COOMBS, W. T. (2006). Today's public relations: An introduction. Sage. Thousand Oaks, CA.

HOLTZHASUSEN, D.; VOTO, R. (2002). "Resistence from the margins: The postmodern public relations practitioner as organizational activits". En: Journal of Public Relations Research, 14, 1, 57-84. 
HUERTAS, A.; ORDEIX, E.; LOZANO, N, (2012). "The excellent communication practice in Spanish organizations. Implementation of Excellence study of $\mathrm{J}$. Grunig in Spain”. En: Ordeix, E. \& Rom, J., Trípodos [EXTRA], 95-114.

INGENHOFF, D.; FUHRER, T. (2010).'Positioning and differentiation by using brand personality attributes. Do mission and vision statements contribute to building a unique corporate identity? Corporate Communications". En: An International Journal, 15,1, 83-101.

LALUEZA, F. (2006). "Does persuasive communication constitute the communicative paradigm of the knowledge society?". En UOC Papers. Journal on Knowledge Society, 3. http://www.uoc.edu/uocpapers/3/dt/eng/lalueza.html. [15-06-2013].

ORDEIX, E.; ROM, J.; BOTEY, J. (2013). "Benchmark Chile-Spain: Qualitative Analysis For Excellence Professional Development of Public Relations In Both Countries". En: GIRALSKI, Margaret A., LEBLANC, H. Paul (eds.). Business Research Yearbook. The Importance of Research to the Global Community, XX, 1, 246-253.

ORDEIX, E., DUARTE, J. (2009). "From Public Diplomacy to Corporate Diplomacy: Increasing Corporation's Legitimacy and Influence". En: American Behavioral Scientist, 53, 4, 549-564.

ORDEIX, E.; ROM, J.. (eds). (2012). "La innovació en Relacions Públiques/La innovación en Relaciones Públicas/Innovation in Public Relations". En: Trípodos Extra 2012.

PAYNE, G. (2009): "President Barack Obama: Advocate of Grassroots Public Diplomacy". En: Trípodos, 24, 1, 11-19.

POTTER, S.; ROBY, H.; COOK, M.; LANGENDAHL, P. (2011). "Transport and Environmental Innovation", Sustainable Innovation 11 'State of the Art in Sustainable Innovation and Design'. University of Surrey, Farnham.

SIMÓ, S.; DE SAN EUGENIO, J.; GINESTA, X. (2012) "El proyecto 'Ecosportech' como ejemplo de universidad emprendedora. Una experiencia a base del 'Problem Based Learning'”. En: Estudios sobre el mensaje periodístico, 18, 2012, p. 869.877.

TOLEDANO, M.;MCKIE,. (2007): "Social Integration and Public Relations: Global lessons from an Israeli Experience". En: Public Relations Review, 37, 387-397.

XIFRA, J.; ORDEIX, E. (2009). "Managing reputational risk in an economic downturn: The case of Banco Santander". En: Public Relations Review, 35, 2009, p. 353-360.

\section{Los autores}

Enric Ordeix Rigo. Doctor en Publicidad y Relaciones Públicas. Profesor Investigador de la Facultad de Comunicación Blanquerna-Universidad Ramon Llull. Miembro del Grupo de Investigación en Estrategia y Creatividad en Publicidad y Relaciones Públicas. 
Director Académico del MA-Strategic Management in Global Communication y del Máster de Protocolo y Relaciones Institucionales. Vicepresidente del Global Communication Institute.

Josep Rom Rodríguez. Doctor en Publicidad y Relaciones Públicas. Profesor titular y vicedecano de la Facultat de Comunicació Blanquerna-Universitat Ramon Llull. Director del Grupo de Investigación en Estrategia y Creatividad en Publicidad y Relaciones Públicas. Director académico de los másters universitarios de Estrategia y Creatividad y Dirección de Arte en Publicidad de la Universitat Ramon Llull.

Jordi Botey López. Doctor en Publicidad y Relaciones Públicas. Profesor Investigador de la Facultad de Comunicación Blanquerna-Universidad Ramon Llull. Miembro del Grupo de Investigación en Estrategia y Creatividad en Publicidad y Relaciones Públicas. Coordinador de Másters y Postgrados (Títulos Propios) de la Facultad de Comunicación Blanquerna-Universidad Ramon Llull. Director Académico del D.E.U. en Comunicació Digital y del D.E.U. en Comunicación en Salud (Healthcare \& Wellness Communication). 